\title{
Why so many published studies and so little progress in necrotizing enterocolitis?
}

In the article Developing necrotizing enterocolitis: retrospective analysis of 1428 preterm infants at a level-III neonatal intensive care unit over a four years period, Ongun et al. conducted a complete retrospective analysis on a complex condition which is almost exclusive to the neonatal intensive care unit (NICU): necrotizing enterocolitis (NEC). This study was conducted in the level-III Division of Neonatology (equivalent to level IIIB in Argentina, as determined by Argentina's Ministry of Health) of Istinye University Faculty of Medicine, Medical Park Hospital, a regional center located in Antalya, a city on the Mediterranean coast, in the southwest of Turkey, with a population of about 2500000 inhabitants.

The study included newborns (NB) younger than 37 weeks of gestation $(<37$ weeks), seen over a 4-year period (2015-2018), and reported the epidemiological and clinical characteristics of the disease.

First of all, I would like to point out that many of my humble remarks are guided by concepts expressed in several papers on the topic by Josef Neu, M.D. (Gainesville, Florida, USA), who has been investigating aspects related to gastrointestinal development and alterations in the neonatal period for 40 years. ${ }^{1}$

When focusing on the article by Ongun et al., my attention was drawn to the high prevalence of NEC in NB < 37 weeks: $18.3 \%$. A recent systematic review of randomized-controlled trials on this topic reported a value of $5-10 \%$ in $\mathrm{NB}<1500 \mathrm{~g}$, ${ }^{2}$ a population that, a priori, could be considered at high risk for this condition.

The high prevalence of NEC in the studied population could be justified by the fact that a neonatal referral center was analyzed, where almost half of NB were transferred from institutions of lower levels of care, probably because of perinatal regionalization recommendations.

Nevertheless, we should ask ourselves what we mean when diagnosing NEC. Bell's radiological criteria were described in $1978^{3}$ and marked this condition for decades because of their usefulness and practicality. In 2007, Gordon et al. ${ }^{4}$ envisaged the need of updating those criteria with a more clinical approach without neglecting the radiological contribution. Ultrasound examination has been recently incorporated to the diagnosis of $\mathrm{NEC}^{5}{ }^{5}$ which is no small feat if we consider its simple access to NB. Nevertheless, its "operator-dependent" modality may interfere with a correct diagnostic interpretation.

The analysis of results for acquired NEC is divided, based on severity, into suspected or grade I in Bell's classification and advanced or grade II or III; in addition, intestinal perforation is confirmed by radiology or surgery.

I believe that the determination of suspected cases (54\% of acquired NEC) may be overdiagnosed; clinical signs such as abdominal distention, gastric residuals and temperature instability, are common findings in the population of preterm NB.

Different interpretations would apply to advanced NEC and intestinal perforation, where better-defined radiological signs (intestinal pneumatosis, portal venous gas or free air in the abdominal cavity) confirm the severity of the clinical features and indications for surgery in most cases.

Ratifying the need for a correct NEC diagnosis, we should bear in mind that there are clinical presentations which frequently create confusion because of their similar symptomatology: ${ }^{6}$

- Spontaneous isolated perforation in the terminal ileum in an antimesenteric location.

- Ischemic intestinal necrosis secondary to heart disease.

- Cow's milk protein-induced enterocolitis (and also breast milk-induced if the mother was exposed to protein intake).

- Congenital megacolon mimicking NEC.

I agree with J. Neu's remarks, ${ }^{6}$ who summarizes NEC as a neonatal disease that progresses rapidly within 24-48 h and distinguishes "medical NEC" with well-defined clinical and radiological signs (intestinal pneumatosis, portal venous gas) and "surgical $N E C^{\prime \prime}$ characterized by intestinal perforation with necrosis seen at surgery or autopsy.

Going back to the presentation by Ongun et al., we should analyze the characteristics of the care provided at the reported division: a highcomplexity level-III division with 34 beds and a nurse-to-patient ratio of 1 nurse per $4-5$ patients in the NICU. There is evidence suggesting that an inadequate nurse-to-patient ratio leads to more healthcare-related adverse events. ${ }^{7}$ 
If we focus our attention on the pathophysiological aspects of NEC, the range of our considerations or hypotheses will be broadened in the face of still unknown data. Probably the intestinal bacterial colonization or the gut microbiome and its changes from fetal to postnatal life are the most interesting aspects to be analyzed when considering the pathogenesis of NEC. Gestational age and mode of delivery have been proposed as determining factors; the high prevalence of $\mathrm{C}$-sections would determine an intestinal microbiota or "dysbiosis" in NB $<1500 \mathrm{~g},{ }^{8}$ together with an increased antibiotic use in the perinatal period.

But maybe one of the most significant factors in disease development is the type of milk and the timing of its first administration. Freshly expressed breast milk plays a critical role in the prevention of NEC. A recent systematic review and meta-analysis showed similar results for breast milk stored in refrigerators and breast milk from milk banks. ${ }^{9}$

The timing of the first administration of breast milk also plays a preventive role: the earlier its administration, with small volumes and slow increases, the higher the immune protection of $\mathrm{NB}<1500 \mathrm{~g}$ or $<32$ weeks of gestation; this finding was demonstrated in this retrospective analysis, where prolonged fasting favored the development of advanced NEC.

Probiotic use may be a valid alternative for the prevention of NEC; nevertheless, its biological characteristics have not yet been defined. Prospective studies with scientific rigor are required to assess its therapeutic effectiveness and patient safety. ${ }^{10}$

Another aspect to be taken into account in the prevention of NEC is the clinical condition the NB should have when attempting enteral feeding; respiratory distress and patent ductus arteriosus (PDA) with hemodynamic instability are associated with advanced grades of NEC in the analysis conducted by Ongun et al. This observation has been reported in the bibliography, despite not being definitely proved. ${ }^{11}$

The high mortality rate $(9 \%)$ observed in the overall population of $\mathrm{NB}<37$ weeks is surprising; the risk of death is 2.1 times higher with NEC development and 11.5 times higher with intestinal perforation. In advanced stages, the mortality rate was $27.3 \%$, and $48.9 \%$ with intestinal perforation. In a broad, prospective, multicenter analysis conducted in a population of NB $<1500 \mathrm{~g}$, Hull M et al. ${ }^{12}$ reported a mortality rate of $21 \%$ among "medical" NEC cases and of $35 \%$ for "surgical" NEC.

Low 5-minute Apgar scores and birth weight $<750 \mathrm{~g}$ are described as risk factors for developing advanced NEC grades and intestinal perforation. These findings are part of this complex and serious disease which, like many others (e.g., retinopathy of prematurity), might represent an overall quality index in peri- and neonatal care (B. Darlow, M.D.).

To sum up, this was an interesting retrospective study with surprising results regarding a condition that still puzzles neonatologists, pediatric surgeons, and researchers. Maybe Josef Neu's question ${ }^{6}$ "Why so many studies and so little progress?" will remain unanswered.

Ernesto Alda, M.D.

Pediatrician and Neonatologist SAP - NAM

Department of Neonatology

Hospital Privado del Sur - Bahía Blanca ROP Argentina Group

http:/ / dx.doi.org/10.5546/ aap.2020.eng.372

To cite: Alda E. Why so many published studies and so little progress in necrotizing enterocolitis? Arch Argent Pediatr 2020;118(6):372-374.

\section{REFERENCES}

1. Stevenson D, Hsu Y, McMorrow M, Berseth C, et al. Component concentrations and activation of the complement system in neonatal illness: a preliminary study of necrotizing enterocolitis. Eur J Pediatr. 1980;134(3):255-9.

2. Xiong T, Maheshwari A, Neu J, EI-Saie A, Pammi M. An Overview of Systematic Reviews of RandomizedControlled Trials for Preventing Necrotizing Enterocolitis in Preterm Infants. Neonatology. 2020;117(1):46-56.

3. Bell MJ, Ternberg JL, Feigin RD, Marshall R, et al. Neonatal necrotizing enterocolitis: therapeutic decisions based upon clinical staging. Ann Surg. 1978;187(1):1-7.

4. Gordon PV, Swanson JR, Attridge JT, Clark R. Emerging trends in acquired neonatal intestinal disease: is it time to abandon Bell's criteria? J Perinatol. 2007;27(11):661-71.

5. Kim JH. Role of abdominal US in diagnosis of NEC. Clin Perinatol. 2019; 46(1):119-27.

6. Neu J. Necrotizing Enterocolitis: The Future. Neonatology. 2020;117(2):240-4.

7. Beltempo M, Lacroix G, Cabot M, Blais R, Piedboeuf B. Association of nursing overtime, nurse staffing and unit occupancy with medical incidents and outcomes of very preterm infants. J Perinatol. 2017;38(2):175-80.

8. Kim C, Claud E. Necrotizing enterocolitis pathophysiology: how microbiome data alters our understanding. Clin Perinatol. 2019;46(1):29-38.

9. Altobelli E, Angeletti P, Verrotti A, Petrocelli R. The Impact of Human Milk on Necrotizing Enterocolitis: A Systematic Review and Meta-Analysis. Nutrients. 2020;12(5):E1322.

10. Lenfestey MW, Neu J. Probiotics in newborns and children. 
374 / Arch Argent Pediatr 2020;118(6):372-374 / Editorial comment

Pediatr Clin North Am. 2017;64(6):1271-89.

11. Kessler U, Schulte F, Cholewa D, Nelle M, et al. Outcome in neonates with necrotizing enterocolitis and patent ductus arteriosus. World J Pediatr. 2016;12(1):55-9.
12. Hull M, Fisher J, Gutierrez I, Jones B, et al. Mortality and management of surgical necrotizing enterocolitis in very low birth weight neonates: a prospective cohort study. J Am Coll Surg. 2014;218(6):1148-55. 